\title{
Impact of sleep behavior on glycemic control in type 1 diabetes: the role of social jetlag
}

\author{
Sandra Larcher1, Anne-Sophie Gauchez 2,3, Sandrine Lablanche ${ }^{1,4}$, \\ Jean-Louis Pépin ${ }^{5,6}$, Pierre-Yves Benhamou' and Anne-Laure Borel ${ }^{1,5}$
}

${ }^{1}$ Grenoble Alpes University Hospital, Pole DIGIDUNE, Department of Endocrinology, Grenoble, France, ${ }^{2}$ Grenoble Alpes University Hospital, Pole biology, "Institut de Biologie et de Pathologie", Grenoble, France, ${ }^{3}$ UMR-S INSERM 1039, " "Laboratoire de bioénergétique fondamentale et appliquée”, INSERM U1055, ${ }^{5}$ Hypoxia Pathophysiology Laboratory, INSERM U1042, Grenoble Alpes University, Grenoble, France, and ${ }^{6}$ Grenoble Alpes University Hospital, Pole Thorax et Vaisseaux, Physiology, Sleep and Exercise Clinic, Grenoble, France

\author{
Correspondence \\ should be addressed \\ to A-L Borel \\ Email \\ alborel@chu-grenoble.fr
}

\begin{abstract}
Background: Sleep behavior is changing toward shorter sleep duration and a later chronotype. It results in a sleep debt that is acquitted on work-free days, inducing a small but recurrent sleep misalignment each week, referred to as "social jetlag". These sleep habits could affect health through misalignment with circadian rhythms.

Objectives: The primary objective is to address the impact of sleep behavior on glycemic control, assessed by HbA1c, in patients with type 1 diabetes, independently of other lifestyle or sleep-related factors. The secondary objective is to address whether circadian phase affects glycemic control.

Design: In total, 80 adult patients with type 1 diabetes ( $46 \%$ female) were included in a clinical cohort study. Methods: Sleep behavior was addressed objectively by a 7-day actimetry, lifestyle by questionnaires, sleep breathing disorders by nocturnal oximetry and circadian phase by dim light melatonin onset (DLMO).

Results: Univariate analyses showed that chronotype $(r=0.23, P=0.042)$ and social jetlag $(r=0.30, P=0.008)$ were significantly associated with $\mathrm{HbA} 1 \mathrm{c}$. In multivariable analysis, social jetlag was the only sleep habit independently associated with $\mathrm{HbA1c}(\beta=0.012(0.006 ; 0.017), P<0.001)$. HbA1c was lower in patients with a social jetlag below versus above the median $(7.7 \%(7.1-8.7)$ and $8.7 \%$ (7.6-9.8), $P=0.011)$. DLMO was not associated with HbA1c. However, the later the DLMO, the worse the sleep efficiency $(r=-0.41, P<0.001)$ and fragmentation index $(r=0.35, P=0.005)$. Conclusions: Social jetlag, a small but recurrent circadian misalignment, is associated with worse glycemic control in type 1 diabetes, whereas circadian phase is not. Further intervention studies should address the potential improvement of glycemic control by correcting social jetlag.

\section{Introduction}

Sleep behavior in the general population is changing toward shorter sleep duration and a later chronotype (i.e., the tendency to go to bed and wake up later) (1). Having a late chronotype but with wake-up time under the constraints of a social schedule leads to a reduction in sleep duration on working days. This results in a sleep debt that is acquitted on work-free days, inducing a small but recurrent sleep misalignment each week, between working and free days. This misalignment has been referred to as "social jetlag" (2).
C 2016 European Society of Endocrinology Printed in Great Britain
Such societal changes lead to adverse health outcomes. In patients with diabetes, the impact of sleep behavior on metabolic health has received increasing attention (3). Patients with type 1 diabetes, who slept less than $6.5 \mathrm{~h}$ a night, have poorer glycemic control (4). In addition, patients with type 2 diabetes who present with a late chronotype have a worse glycemic control, independent of body weight (5).

However, sleep behavior is likely to be associated with other lifestyle factors that could be confounders for

Published by Bioscientifica Ltd. 
any association between sleep behavior and glycemic control. The social gradient of health is known to affect outcomes of patients with type 1 diabetes (6). Conversely, socioeconomic status (7) and workplace social capital (job demand, job control and social support at work) are known to affect sleep behavior (8). In addition, sleep behavior might also affect eating habits (9). These eating habits as well as sleep diseases could be potential confounders for the relationship between sleep behavior and glycemic control.

The main objective of the study is to evaluate which specific sleep behavior, that is, sleep duration, sleep debt, chronotype or social jetlag, might be related to glycemic control independently of other lifestyle or sleep-related factors.

The second objective of the study is to evaluate whether circadian phase and misalignment between circadian phase and social time may interact with glycemic control.

\section{Subjects and methods}

A total of 81 patients with type 1 diabetes were prospectively recruited from the outpatient clinic and from educational programs in Grenoble University Hospital (France) between January 2014 and November 2014. One patient dropped-out for personal reasons immediately after inclusion. Thus, 80 patients were analyzed. See Fig. 1 for flowchart. The inclusion criteria for the study were adult patients with type 1 diabetes, without upper limit for age. The exclusion criteria were pregnancy, acute diabetesrelated disorder (severe hypoglycemia or acidoketosis) within the last 3 months and shift work. Sleep behavior has been assessed objectively by a 7-day wrist actimetry. The other lifestyle factors were carefully collected

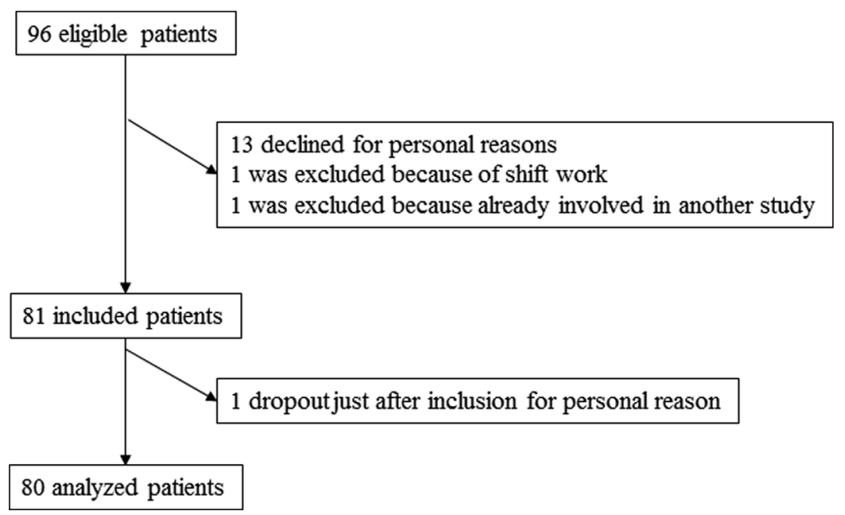

Figure 1

Flowchart. (socioeconomic status, job strain and dietary habits). Sleep breathing disorders were screened by nocturnal oximetry. Circadian phase was estimated by dim light melatonin onset (DLMO). The main outcome was glycemic control, as estimated by glycated hemoglobin.

\section{Study design: clinical cohort study}

Patients underwent a medical examination and anthropometric measurements by the same investigator (SL) who also collected their medical history and the following data. Each patient-signed consent was obtained after full explanation of the purpose and nature of all procedures used. The study was approved by the ethics committee Sud-Est V (IRB 6705).

\section{Questionnaires}

Patients were asked about their familial status (living alone or living as a couple, number of children), level of education and employment status. Precarity was assessed by the "Evaluation de la Précarité et des Inégalités de santé dans les Centres d'Examens de Santé" (EPICES) questionnaire (10). This is a French questionnaire (Assessment of insecurity and health Inequalities in Health Examination Centers) giving a continuous score ranging between 0 (absence of precarity) and 100 (highest level of precarity) with a threshold set at 30 .

Job strain was assessed by the KARASEK questionnaires. The demand-control model proposed by KARASEK defines job strain as a consequence of the combination of high psychological job demands and low job decision latitude. The patients are classified into three conditions: (i) without job strain, (ii) with job strain and (iii) with job strain and social isolation at work (11).

The 24-h dietary recall was realized by interview and was unexpected by patients as it was performed on the day of inclusion. Patients reported the timing, content and quantity of food consumption during the last $24 \mathrm{~h}$. Evening collation refers to a small quantity of food taken after dinner, in a later and separate time than dinner (12).

\section{Wrist actimetry}

The Actiwatch-AW7 (Cambridge Neurotechnology Ltd, Cambridge, UK) is an actigraph measuring activity by means of a piezoelectric accelerometer that records the combination of intensity and amount and duration of movement, and the corresponding voltage produced is converted and stored as an activity count. The software 
(Actiwatch Activity and Sleep analysis 7.31; CamNtech Ltd) was set to detect activity with "medium" sensitivity, that is, 40 counts per epoch. The event marker button on top of the Actiwatch had to be pressed at bedtime and getup time. The sleep analysis software determines sleep start by searching for a period of at least $10 \mathrm{~min}$ of consecutively recorded immobile data following bedtime. To determine the end of sleep, the software analyzed the end of immobility corresponding to the getup time event (13). In addition, a self-reported sleep diary was completed by participants to determine bedtime, subjective sleep latency, wake-up and getup time, as well as number of awakenings during the night, as recommended (14). The Actiwatch-AW7 was worn on the wrist for seven consecutive days. In addition to sleep duration, sleep latency (i.e., time to get to sleep after going to bed), sleep fragmentation index (i.e., total number of awakenings divided by total sleep time) and sleep efficiency (i.e., percentage of time spent asleep reported on time spent in bed) were automatically analyzed by the actimetry software. These measurements have been validated to be similar to those measured by the gold standard, polysomnography (15). Sleep debt was calculated as the absolute value of mean sleep duration per night during weekends (Friday and Saturday nights) minus mean sleep duration per night during the week $(5,16)$. Chronotype was calculated as the mid-sleep time (midpoint between sleep onset and wake-up time) on free days (MSF) with further correction for sleep debt as follows: Chronotype $=$ MSF $-0.5 \times$ sleep debt (16). Social jetlag, defined as the circadian misalignment induced by a social schedule (2), was calculated as the absolute difference between mid-sleep time on free days and week days $($ MSW $)=\mid$ MSW - MSF $\mid$. This definition was also used for unemployed subjects taking into account that social constraints might be different between week and weekend, based on children or non-working activities. Finally, it has been shown that season of assessment could have an effect on sleep duration, chronotype and social jetlag (17). Thus, season of assessment was taken into account, described as "daylight saving time" for the summer period where social time is 1-h in advance (end of March to end of October) and "standard time" for the winter period (end of October to end of March) (17).

\section{Nighttime pulse oximetry}

Patients were screened for obstructive sleep apnea by nocturnal oximetry with NONIN 2500 finger pulse oximeter (Nonin Medical Inc, Minneapolis, MN, USA). Threshold was $3 \%$ oxygen desaturation index (ODI) above 10 events/h (18).

\section{Biological measurements}

Melatonin concentrations were measured from saliva samples collected every $30 \mathrm{~min}$, starting from 19:30 until 22:30. DLMO usually occurs within this time interval (19). Participants were allowed to drink only water and had no fluids for $25 \mathrm{~min}$ before each sample collection. Saliva was collected under dim light conditions ( $<50$ lux) defined as a room with lights out expect for a small, distant light or TV screen. The radioimmunoassay had an intra-assay precision of $<5 \%$ and an inter-assay precision of $7.0 \%$ (Melatonin direct Saliva RIA IBL International GMBH, Flughafenstrasse 52a D-22335 Hamburg, Germany). The limit of detection for this assay was $0.3 \mathrm{pg} / \mathrm{mL}$. For some time points with inadequate saliva volume, determination of melatonin concentrations was not possible. Patients were included in melatonin analyses if they had at least four out of the seven possible measurements. Where melatonin production is low, the definition of DLMO depends on baseline data availability (20), which we did not have. Therefore, to use an objective criterion, the 30-min epoch in which the greatest rise in melatonin occurred was retained to define DLMO. For statistical analysis, the time at the end of this epoch was chosen as the DLMO, as proposed previously (21).

HbA1c was measured to reflect 3-month glycemic control, unaffected by acute variation of plasmatic glycemia (high-pressure liquid chromatography, Varian II; Bio-Rad). Microalbuminuria was measured by immunoturbidimetry (Nephelometer Analyser II; Behring, Marburg, Germany). Plasma HDL-cholesterol, triglycerides, LDL-cholesterol and creatinine levels were determined according to standardized procedures $(22,23,24)$.

\section{Estimated glucose disposal rate (eGDR)}

To estimate insulin sensitivity, eGDR was calculated as follows: eGDR $(\mathrm{mg} / \mathrm{kg} / \mathrm{min})=21.158+(-0.09 \times$ waist circumference $)+(-3.407 \times$ hypertension $)+(-0.551 \times \mathrm{HbA} 1 \mathrm{c}) \quad$ as validated $(25,26)$.

\section{Statistical analysis}

Sample size was estimated based on a previous study (4).

The normal distribution of variables was verified by the Shapiro-Wilk test. Skewed variables that were not normalized despite mathematical transformation were then tested by non-parametric tests where necessary. 
Sleep behavior parameters were extracted from actimetry when available and from the sleep diary when actimetric data were missing $(n=4)$, as recommended (14). Other missing values (always below 10\%) were replaced by medians.

Main objective: Crude associations with HbA1c were tested by Pearson's or Spearman's correlations, for normally and not normally distributed variables respectively. ANOVA tests were used to address association

Table 1 Characteristics of subjects. Data are presented as mean (s.D.) if normally distributed or median (IQR) if not normally distributed. Qualitative variables are presented by frequency.

\begin{tabular}{|c|c|}
\hline & $n=80$ \\
\hline Age (years) & $41(27-51)$ \\
\hline Female $(n \%)$ & $37(46)$ \\
\hline BMI $\left(\mathrm{kg} / \mathrm{m}^{2}\right)$ & $24.4(3.8)$ \\
\hline \multicolumn{2}{|l|}{ Characteristics of diabetes } \\
\hline Diabetes duration (years) & $13.75(9.25-24.50)$ \\
\hline Daily insulin doses (IU) & $44(19)$ \\
\hline eGDR $(\mathrm{mg} / \mathrm{kg} / \mathrm{min})$ & $8.68(6.41-9.62)$ \\
\hline $\mathrm{HbA} 1 \mathrm{c}(\%)$ & $8.3(1.5)$ \\
\hline $\mathrm{HbA} 1 \mathrm{c}(\mathrm{mmol} / \mathrm{mol})$ & $67.2(12.1)$ \\
\hline Retinopathy $(n \%)$ & $24(30)$ \\
\hline Nephropathy (n \%) & $8(11)$ \\
\hline Sensitive neuropathy $(n \%)$ & $9(11)$ \\
\hline Vegetative neuropathy ( $n \%$ ) & $4(5)$ \\
\hline Cardiac autonomic neuropathy & $10(13)$ \\
\hline Foot ulceration ( $n$ \%) & $3(4)$ \\
\hline \multicolumn{2}{|l|}{ Other cardiovascular risk factors } \\
\hline \multicolumn{2}{|l|}{ Tobacco consumption } \\
\hline None/former & $56(70)$ \\
\hline Current & $24(30)$ \\
\hline Hypertension (n \%) & $20(25)$ \\
\hline Dyslipidemia $(n \%)$ & $20(25)$ \\
\hline $\mathrm{HDL}-\mathrm{C}(\mathrm{mmol} / \mathrm{L})$ & $1.57(0.43)$ \\
\hline LDL-C (mmol/L) & $2.53(0.88)$ \\
\hline Triglycerides (mmol/L) & $0.89(0.72-1.19)$ \\
\hline \multicolumn{2}{|l|}{ Oximetry } \\
\hline ODI (events/h) & $2.40(1.57-5.64)$ \\
\hline ODI>10 (n \%) & $9(11)$ \\
\hline \multicolumn{2}{|l|}{ Actimetry } \\
\hline Sleep duration (min) & $458(53)$ \\
\hline Sleep debt (min/week) & $60(25-96)$ \\
\hline Chronotype (h:min) & 04:03 (03:37-04:43) \\
\hline Social jetlag (min) & $49(24-79)$ \\
\hline \multicolumn{2}{|l|}{ Distribution of caloric intake (\%) } \\
\hline Breakfast & $14.9(2.2-24.0)$ \\
\hline Lunch & $44.6(35.7-51.4)$ \\
\hline Dinner & $38.3(30.6-45.7)$ \\
\hline Late evening collation ( $n$ \%) & $5(6)$ \\
\hline Coffee (units/d) & $2(1-4)$ \\
\hline Tea (units/d) & $0(0-2)$ \\
\hline
\end{tabular}

BMI, body mass index; eGDR, estimated glucose disposal rate; HDL, high-density lipoprotein; LDL, low-density lipoprotein; ODI, oxygen desaturation index.
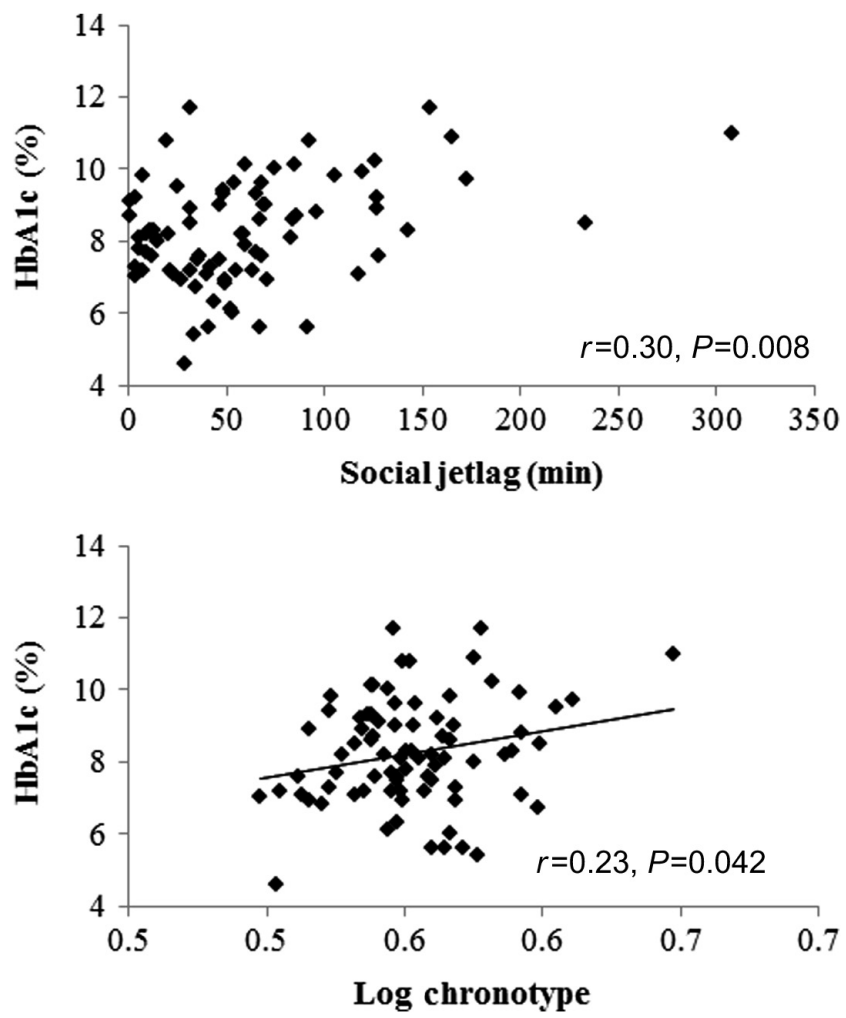

Figure 2

Univariate correlations between social jetlag (Spearman's correlation), Log chronotype (Pearson's correlation) and HbA1c.

between qualitative variables and HbA1c. All univariate associations that demonstrated a $P$-value below 0.2 for their link to HbA1c were then entered in a multivariable

Table 2 Relative contribution toward the variance of $\mathrm{HbA} 1 \mathrm{c}$. Analyses were made by a multiple variable forward regression model in which all variables associated with a $P$-value below 0.2 in univariate analyses were entered. Marital status was separated between married vs single and divorced vs single.

\begin{tabular}{l}
\hline \\
\hline Model 1 \\
Adjusted $R^{2}=42 \%$ \\
Daily insulin dose \\
Social jetlag \\
Lunch caloric intake \\
Lunch time \\
Coffee \\
Evening collation \\
Tobacco consumption \\
Living as a couple vs \\
living alone
\end{tabular}

\begin{tabular}{ccc}
\hline $\boldsymbol{\beta}$-coefficient $(95, \mathrm{Cl})$ & & $\boldsymbol{P}$-value \\
\cline { 1 - 1 } $0.011(-0.003 ; 0.025)$ & & 0.111 \\
$0.012(0.006 ; 0.017)$ & & $<\mathbf{0 . 0 0 1}$ \\
$-0.014(-0.037 ; 0.008)$ & & 0.203 \\
$-0.006(-0.011 ; 0.000)$ & & 0.057 \\
$0.168(0.039 ; 0.297)$ & & $\mathbf{0 . 0 1 1}$ \\
$1.780(0.686 ; 2.874)$ & & $\mathbf{0 . 0 0 2}$ \\
$0.903(0.286 ; 1.520)$ & & $\mathbf{0 . 0 0 5}$ \\
$-0.580(-1.143 ;-0.017)$ & $\mathbf{0 . 0 4 4}$ \\
\hline
\end{tabular}

P-values in bold are statistically significant $(<0.05)$. 
forward regression model with $\mathrm{HbA1c}$ as a dependent variable. Collinearity between sleep variables was assessed before entering independent variables into the multivariable model. The variables being specific and collinearity acceptable $(r \leq 0.41)$, only one model including all sleep variables was built.

Characteristics of patients were then compared by Wilcoxon tests and $\chi^{2}$ tests (Fisher's test if $n<5$ ) between those having a small and those having a larger social jetlag, separated by the median of social jetlag.
Secondary objective: melatonin measurements were missing in 16 patients out of 80 (20\%). Thus, analyses of melatonin-related variables were conducted on 64 patients. DLMO was examined for its potential association with $\mathrm{HbA1c}$ as well as with sleep-related variables.

\section{Results}

Characteristics of patients are summarized in Table 1. Of the study patients, $65 \%$ were married, 54\% were

Table 3 Comparisons of patient characteristics according to social jetlag. Quantitative variables are presented as median (IQR) and qualitative variables by frequency $(n(\%))$. The differences between groups has been tested by Wilcoxon tests and $\chi^{2}$ tests respectively (Fisher's test if $n<5$ ).

\begin{tabular}{|c|c|c|c|}
\hline & Social jetlag $\leq 49 \min (n=41)$ & Social jetlag > $49 \min (n=39)$ & $P$-value \\
\hline Age (years) & $44(30-51)$ & $38(25-47)$ & 0.065 \\
\hline Female sex $(n(\%))$ & $21(51)$ & $16(41)$ & 0.361 \\
\hline BMI $\left(\mathrm{kg} / \mathrm{m}^{2}\right)$ & $24.0(21.3-27.2)$ & $24.0(22.1-27.5)$ & 0.564 \\
\hline \multicolumn{4}{|l|}{ Characteristics of diabetes } \\
\hline Diabetes duration (years) & $13.00(7.50-25.00)$ & $14.00(10.50-22.00)$ & 0.697 \\
\hline Daily insulin dose (IU) & $37(28-53)$ & $45(35-60)$ & 0.134 \\
\hline eGDR & $8.73(7.72-9.72)$ & $8.58(5.15-9.61)$ & 0.329 \\
\hline $\mathrm{HbA} 1 \mathrm{c}(\%)$ & $7.7(7.1-8.7)$ & $8.7(7.6-9.8)$ & 0.011 \\
\hline $\mathrm{HbA} 1 \mathrm{c}(\mathrm{mmol} / \mathrm{mol})$ & $60.7(54.1-71.6)$ & $71.6(59.6-83.6)$ & 0.011 \\
\hline Retinopathy (n (\%)) & $12(29)$ & $12(31)$ & 0.884 \\
\hline Nephropathy (n (\%)) & $3(8)$ & 5 (15) & 0.472 \\
\hline Sensitive neuropathy (n (\%)) & $4(10)$ & $5(13)$ & 0.734 \\
\hline Vegetative neuropathy $(n(\%))$ & $1(2)$ & $3(8)$ & 0.353 \\
\hline CAN $(n(\%))$ & $3(7)$ & $7(18)$ & 0.188 \\
\hline Foot ulceration (n (\%)) & $1(2)$ & $2(5)$ & 0.611 \\
\hline \multicolumn{4}{|l|}{ Other cardiovascular risk factors } \\
\hline Tobacco consumption & & & 0.036 \\
\hline None & $30(73)$ & $19(49)$ & \\
\hline Current & $10(24)$ & $14(36)$ & \\
\hline Former & $1(2)$ & $6(15)$ & \\
\hline Systolic BP (mmHg) & $124(116-131)$ & $125(116-135)$ & 0.862 \\
\hline Diastolic BP (mmHg) & $68(63-77)$ & $68(63-77)$ & 0.870 \\
\hline Hypertension (n (\%)) & $9(22)$ & $11(28)$ & 0.519 \\
\hline Dyslipidemia (n (\%)) & $13(32)$ & $7(18)$ & 0.156 \\
\hline Triglycerides (mmol/L) & $0.87(0.70-0.90)$ & $0.99(0.80-1.60)$ & 0.004 \\
\hline $\mathrm{HDL}-\mathrm{C}(\mathrm{mmol} / \mathrm{L})$ & $1.57(1.39-1.88)$ & $1.57(1.19-1.82)$ & 0.187 \\
\hline LDL-C (mmol/L) & $2.53(2.01-3.24)$ & $2.53(1.91-2.81)$ & 0.580 \\
\hline \multicolumn{4}{|l|}{ Oximetry } \\
\hline ODI (events/h) & $2.4(1.4-4.9)$ & $2.4(1.9-9.1)$ & 0.279 \\
\hline ODI>10 (n (\%)) & $2(5)$ & 7 (18) & 0.084 \\
\hline \multicolumn{4}{|l|}{ Actimetry } \\
\hline Sleep duration (min) & 460 (427-499) & 456 (433-472) & 0.290 \\
\hline Sleep debt (min/week) & $43(24-69)$ & $76(28-145)$ & 0.036 \\
\hline Chronotype (h:min) & 03:58 (03:15-04:26) & 04:30 (03:51-05:24) & 0.004 \\
\hline Social jetlag (min) & $25(9-36)$ & $83(65-126)$ & $<0.001$ \\
\hline \multicolumn{4}{|c|}{ Distribution of daily caloric intake (\%) } \\
\hline Breakfast & $16.9(9.0-25.3)$ & $12.0(0.0-21.0)$ & 0.176 \\
\hline Lunch & $43.7(38.2-49.0)$ & $44.6(34.0-56.3)$ & 0.551 \\
\hline Dinner & $37.8(31.0-43.0)$ & $39.5(29.3-45.9)$ & 0.668 \\
\hline Evening collation $(n(\%))$ & $4(10)$ & $1(3)$ & 0.361 \\
\hline
\end{tabular}

BMI, body mass index; BP, blood pressure; CAN, cardiac autonomic neuropathy; eGDR, estimated glucose disposal rate; HDL, high-density lipoprotein; LDL, low-density lipoprotein; ODI, oxygen desaturation index. P-values in bold are statistically significant (<0.05). 
graduates, $51 \%$ worked full time, $11 \%$ part time and the others were not working. Working patients had non-flexible schedules in $32 \%$ of cases. Patients were living in precarity in $20 \%$ of cases. For working patients, the KARASEK questionnaire identified 77\% of patients having a non-stressful job, 5\% with job strain and $18 \%$ with job strain and social isolation at work. Sleep parameters were not different according to season assessment.

\section{Main objective: link between sleep behavior and glycemic control}

\section{Univariate associations}

Log of chronotype, social jetlag and tobacco consumption were significantly associated with HbA1c (Fig. 2), whereas sleep debt and sleep duration were not; two patients had social jetlag above $200 \mathrm{~min}$ and thus could have undue amounts of leverage with social jetlag. Analyses were repeated after removing these two outliers without modifying the link between social jetlag and HbA1c $(r=0.27, P=0.017)$.

\section{Multivariable regression}

Social jetlag, coffee consumption, evening collation, tobacco consumption and living alone (vs living as a couple were independently associated with greater HbA1c and explain $42 \%$ of HbA1c variance (Table 2). The independent association of social jetlag was not changed by removing the two outliers who presented with social jetlag above 200 min ( $\beta$-coefficient $(95, \mathrm{CI}) 0.013$ (0.006; $0.020), P<0.001)$. Stratification of analyses between active and inactive patients did not alter the link between social jetlag and glycemic control (data not shown).

\section{Comparison of patients with a small or a large social jetlag}

Patients were divided into two groups according to the median of social jetlag (Table 3). The HbA1c was higher in patients with a larger social jetlag than in those with a small social jetlag. Patients with a larger social jetlag also had a later chronotype and a longer sleep debt. They were more often smokers, their triglyceride levels were higher and they tended to be younger $(P=0.065)$. They did not differ for socioeconomic variables. Neither daily insulin dose nor estimated glucose disposal rate were different between the two groups.

\section{Secondary objective: link between DLMO and glycemic control}

Out of 80 patients, 16 had more than three missing samples and thus were not analyzed. Of them, 44 had all seven samples available. Also, 11, six and three patients had only one sample, two and three out of seven missing respectively. DLMO was inversely correlated with age $(r=-0.25, P=0.045)$. Men had a later DLMO than women $(21.5 \mathrm{~h}$ vs $21.0 \mathrm{~h}, P=0.032$ respectively). Patients assessed during "daylight saving time" (summer) had a 30-min later DLMO than patients assessed during "standard time" (winter) (21.5 (21.0-22.0) vs $21.0 \quad(20.5-21.5) \quad h, \quad P=0.015$ respectively). DLMO did not correlate with HbA1c. There was no significant interaction between DLMO and season of assessment for the relationship with HbA1c. DLMO was not associated with chronotype, social jetlag or sleep duration. However, DLMO was positively correlated with sleep fragmentation $(r=0.35$, $P=0.005)$ and inversely correlated with sleep efficiency $(r=-0.41, P<0.001)$.

We compared HbA1c levels of patients who were concordant for early DLMO and early chronotype (or late DLMO and late chronotype) with those discordant between DLMO and chronotype. We found no difference between DLMO/chronotype concordant or discordant groups for HbA1c.

\section{Discussion}

Social jetlag was the only sleep characteristic independently associated with glycemic control in patients with type 1 diabetes. The social constraints of early work schedules lead to an increasing sleep debt over the week that is compensated for on weekends. This social jetlag is therefore longer in subjects with later chronotypes. Chronotype is characterized by age and gender specificities: men and younger people more often have a late chronotype. Subjects with late chronotypes, hence more social jetlags, are more likely to smoke, to consume alcohol and coffee and to be depressed (27). Our results were consistent with these previous findings as patients with longer social jetlags had later chronotypes, higher sleep debts, tended to be younger and were also more likely to be active smokers.

Social jetlag is known to be linked to excess body weight and metabolic syndrome $(2,28)$. In this study, patients with short or long social jetlag were not different for body weight. However, patients with longer social 
jetlag had higher levels of triglycerides, a component of metabolic syndrome and a marker of insulin resistancerelated dyslipidemia (29). However, insulin requirement and eGDR, both indirect markers of insulin resistance in patients with type 1 diabetes $(25,26)$, were not different between patients with small and large social jetlag.

In patients with type 2 diabetes, late chronotype was associated with poor glycemic control, possibly mediated by specific food habits (5). Indeed, patients with type 2 diabetes with late chronotype tended to preferentially consume most of their daily caloric intake at the evening meal. In this study, the habits of eating a late evening collation and drinking large quantities of coffee were linked to higher levels of HbA1c. However, their contributions to HbA1c variance were independent from the contribution of social jetlag.

In a previous work, we have demonstrated that patients who slept less than $6.5 \mathrm{~h}$ a night had a higher HbA1c than patients who slept longer (4). In this study, the 7-day mean sleep duration (minimum $5 \mathrm{~h}$ 14 , maximum $10 \mathrm{~h} \mathrm{15)} \mathrm{was} \mathrm{analyzed} \mathrm{as} \mathrm{a} \mathrm{continuous}$ variable and was not associated with HbA1c in univariate analysis. In the present sample of patients, only seven patients $(8.75 \%)$ presented with sleep duration below $6.5 \mathrm{~h}$. Their median HbA1c was 9.0\% (7.6-11.0), whereas the remaining 73 patients had a median $\mathrm{HbA1c}$ of $8.2 \%$ (7.2-9.2), $P=0.236$. The lack of association between sleep duration and HbA1c in this study could be due to the small number of patients with short sleep duration and to a threshold effect in association between sleep duration and glycemic control.

The social gradient in health status is well documented, the lower the socioeconomic position, the worse the health of an individual (30). In addition, job strain (high job demands combined with low personal control) has been shown to affect health, favoring the occurrence of metabolic syndrome (31). In this study, neither socioeconomic status (EPICES score) nor job strain (KARASEK questionnaire) was associated with HbA1c, allowing to exclude these factors as potential confounders of the link between sleep behavior and glycemic control. Nevertheless, we cannot conclude that social status or job strain do not affect glycemic control in patients with type 1 diabetes, as this study was underpowered to address this question.

Our team (32) and others (33) have suggested that sleep apnea syndrome is unexpectedly frequent in patients with type 1 diabetes, who are not obese. In these pilot studies, HbA1c was not clearly associated with the presence of sleep apnea; however, some data suggest that arousal index, assessed by polysomnography, could be linked to glycemic control (34). We therefore included an oximetric recording in the patients' sleep evaluation. With regard to the small number of patients suspected of sleep apnea (11\%), ODI was not linked to $\mathrm{HbA1c}$.

In this study, DLMO was assessed under home conditions instead of controlled conditions of a laboratory. However, a good correlation between in-lab and at-home DLMO assessment has been demonstrated (35). Moreover, at-home DLMO is used in routine clinical care in large cohorts of patients (36). This measure was available for $80 \%$ of patients in our cohort, which is the usual rate of success of such a measurement (36).

Melatonin level usually rises approximately $2 \mathrm{~h}$ before habitual sleep onset. As expected, we found that DLMO becomes earlier with advancing age and that men had a later DLMO than women, as observed previously (27). It was also expected that DLMO would be later during the "saving daylight time" period (summer) than during the "standard time" period (winter) (17). However, in our study, DLMO was not correlated with the chronotype measured by actimetry, whereas previous laboratory sleep studies have shown a robust association between DLMO and time of falling asleep $(37,38,39)$. Our explanation is that DLMO reflected the patients' biological circadian phase that did not correspond to their routine, at-home sleep behavior, suggesting a strong impact of social behavior on sleep timing. Circadian clocks are organized hierarchically, with the central clock localized in the hypothalamus suprachiasmatic nucleus and peripheral clocks localized in other brain regions and peripheral tissues. The central clock is implicated to direct both behavioral and metabolic adaptations leading to a circadian regulation of food intake. One important mediator between central and peripheral clocks is melatonin. Beyond lightdarkness signals, central clock might be determined by polygenic factors, age or sex (3). Where biological time might be in conflict with social schedule, one could expect changes in feeding behavior and metabolic dysregulation. For instance, in the Nurses' Health Study, participants with lower melatonin secretion had a significantly higher incidence of type 2 diabetes (40). In this study, no specific circadian phase was associated with a poorer glycemic control. We also tested whether patients whose biological circadian phase concurred with their socially induced chronotype had better glycemic control than patients who were discordant, but $\mathrm{HbA1c}$ was not different between these two groups. 
The later the DLMO, the worse the sleep efficiency and fragmentation index, suggesting that having a late circadian phase is associated with poor sleep quality. To the best of our knowledge, we did not find other data supporting the same observation. These results need to be confirmed by further studies.

\section{Weaknesses of the study}

The study is observational in nature, conclusions as to causative relationships cannot be drawn from the present work. Several potential confounding factors such as therapeutic observance, timing in insulin injections or hypoglycemic events that could drive the link between sleep behavior and HbA1c have not been collected. In addition, we did not collect information regarding physical activity and its timing. Desirability bias could have occurred in self-reported food intake or because participants could have changed their sleep behavior due to the knowledge that this behavior was recorded.

\section{Conclusion}

This study provides a detailed insight into the sleep behavior of patients with type 1 diabetes and the potential impact on glycemic control. Social jetlag appears as the major factor that alters $\mathrm{HbA1c}$, although the direction of the relationship cannot be extrapolated from the present cross-sectional analysis. Whereas the biological circadian phase was dissociated from the "socially induced" chronotype, suggesting a strong impact of social schedule on sleep timing, this observation was not linked to HbA1c. Further intervention studies should address the potential improvement of glycemic control by reducing social jetlag. Strategies could be either educational, drug or technological interventions that target phase-delayed disorders or interventions that will adapt social schedule to biological circadian phase instead of the opposite.

\section{Declaration of interest}

All authors state that there is no conflict of interest that could be perceived as prejudicing the impartiality of the research reported.

\section{Funding}

This study was funded by Association Nationale pour les Traitements A Domicile, les Innovations et la Recherche and Société Francophone de Diabétologie, Fondation du souffle-comité national contre les maladies respiratoires, Fond Agir pour les maladies chroniques and the Resmed Foundation.

\section{Authors' contribution statement}

S Lar participated in the study design, recruited patients, collected data, participated in writing the manuscript and gave final approval of the version to be published. A S G collected the data, reviewed/edited the manuscript and gave final approval of the version to be published. S Lab and $J L P$ reviewed/edited the manuscript and gave final approval of the version to be published. P Y B recruited patients, reviewed/edited the manuscript and gave final approval of the version to be published. A L B designed the study, collected the data, wrote the manuscript and gave final approval of the version to be published.

\section{Acknowledgements}

The authors thank Nathalie Arnol, professional statistician, for statistical support and Dr Alison Foote (Grenoble University Hospital, Clinical Research Center) for critically reading and editing the manuscript with respect to English language usage.

\section{References}

1 Roenneberg T, Kuehnle T, Juda M, Kantermann T, Allebrandt K, Gordijn M \& Merrow M. Epidemiology of the human circadian clock. Sleep Medicine Reviews 200711 429-438. (doi:10.1016/j. smrv.2007.07.005)

2 Roenneberg T, Allebrandt KV, Merrow M \& Vetter C. Social jetlag and obesity. Current Biology 201222 939-943. (doi:10.1016/ j.cub.2012.03.038)

3 Larcher S, Benhamou PY, Pepin JL \& Borel AL. Sleep habits and diabetes. Diabetes \& Metabolism 201541 263-271. (doi:10.1016/ j.diabet.2014.12.004)

4 Borel AL, Pepin JL, Nasse L, Baguet JP, Netter S \& Benhamou PY. Short sleep duration measured by wrist actimetry is associated with deteriorated glycemic control in type 1 diabetes. Diabetes Care 2013 36 2902-2908. (doi:10.2337/dc12-2038)

5 Reutrakul S, Hood MM, Crowley SJ, Morgan MK, Teodori M, Knutson KL \& Van Cauter E. Chronotype is independently associated with glycemic control in type 2 diabetes. Diabetes Care 201336 2523-2529. (doi:10.2337/dc12-2697)

6 Berhan YT, Eliasson M, Mollsten A, Waernbaum I, Dahlquist G \& Swedish Childhood Diabetes Study Group. Impact of parental socioeconomic status on excess mortality in a population-based cohort of subjects with childhood-onset type 1 diabetes. Diabetes Care 201538 827-832. (doi:10.2337/dc14-1522)

7 Grandner MA, Knutson KL, Troxel W, Hale L, Jean-Louis G \& Miller KE. Implications of sleep and energy drink use for health disparities. Nutrition Reviews 201472 (Supplement 1) 14-22. (doi:10.1111/nure.12137)

8 Takahashi M, Tsutsumi A, Kurioka S, Inoue A, Shimazu A, Kosugi Y \& Kawakami N. Occupational and socioeconomic differences in actigraphically measured sleep. Journal of Sleep Research 201423 458-462. (doi:10.1111/jsr.12136)

9 Reutrakul S, Hood MM, Crowley SJ, Morgan MK, Teodori M \& Knutson KL. The relationship between breakfast skipping, chronotype, and glycemic control in type 2 diabetes. Chronobiology International 201431 64-71. (doi:10.3109/07420528.2013.821614)

10 Bihan H, Laurent S, Sass C, Nguyen G, Huot C, Moulin JJ, Guegen R, Le Toumelin P, Le Clesiau H, La Rosa E et al. Association among individual deprivation, glycemic control, and diabetes complications: the EPICES score. Diabetes Care 200528 2680-2685. (doi:10.2337/ diacare.28.11.2680)

11 Karasek R, Brisson C, Kawakami N, Houtman I, Bongers P \& Amick B. The Job Content Questionnaire (JCQ): an instrument for internationally comparative assessments of psychosocial job 
characteristics. Journal of Occupational Health Psychology 19983 322-355. (doi:10.1037/1076-8998.3.4.322)

12 Le Moullec N, Deheeger M \& Preziosi P. Validation of the photo manual used for the collection of dietary data in the SU.VI.MAX. study. Cahiers de nutrition et de diététique 199631 158-164.

13 Tryon WW. Issues of validity in actigraphic sleep assessment. Sleep 200427 158-165.

14 Littner M, Kushida CA, Anderson WM, Bailey D, Berry RB, Davila DG, Hirshkowitz M, Kapen S, Kramer M, Loube D et al. Practice parameters for the role of actigraphy in the study of sleep and circadian rhythms: an update for 2002. Sleep 200326 337-341.

15 Sadeh A. The role and validity of actigraphy in sleep medicine: an update. Sleep Medicine Reviews 201115 259-267. (doi:10.1016/ j.smrv.2010.10.001)

16 Roenneberg T, Wirz-Justice A \& Merrow M. Life between clocks: daily temporal patterns of human chronotypes. Journal of Biological Rhythms 200318 80-90. (doi:10.1177/0748730402239679)

17 Allebrandt KV, Teder-Laving M, Kantermann T, Peters A, Campbell H, Rudan I, Wilson JF, Metspalu A \& Roenneberg T. Chronotype and sleep duration: the influence of season of assessment. Chronobiology International 201431 731-740. (doi:10.3109/07420528.2014.901347)

18 Series F, Kimoff RJ, Morrison D, Leblanc MH, Smilovitch M, Howlett J, Logan AG, Floras JS \& Bradley TD. Prospective evaluation of nocturnal oximetry for detection of sleep-related breathing disturbances in patients with chronic heart failure. Chest 2005127 1507-1514. (doi:10.1378/chest.127.5.1507)

19 Gooneratne NS, Metlay JP, Guo W, Pack FM, Kapoor S \& Pack AI. The validity and feasibility of saliva melatonin assessment in the elderly. Journal of Pineal Research 200334 88-94. (doi:10.1034/j.1600079X.2003.02945.x)

20 Van Someren EJ \& Nagtegaal E. Improving melatonin circadian phase estimates. Sleep Medicine 20078 590-601. (doi:10.1016/ j.sleep.2007.03.012)

21 Papaioannou I, Twigg GL, Kemp M, Roughton M, Hooper J, Morrell MJ \& Polkey MI. Melatonin concentration as a marker of the circadian phase in patients with obstructive sleep apnoea. Sleep Medicine 201213 167-171. (doi:10.1016/j.sleep.2011.01.020)

22 Burstein M \& Samaille J. On a rapid determination of the cholesterol bound to the serum alpha- and beta-lipoproteins. Clinica Chimica Acta 19605 609. (doi:10.1016/0009-8981(60)90075-9)

23 Friedewald WT, Levy RI \& Fredrickson DS. Estimation of the concentration of low-density lipoprotein cholesterol in plasma, without use of the preparative ultracentrifuge. Clinical Chemistry 1972 18 499-502.

24 Peake M \& Whiting M. Measurement of serum creatinine--current status and future goals. Clinical Biochemist Reviews 200627 173-184.

25 Williams KV, Erbey JR, Becker D, Arslanian S \& Orchard TJ. Can clinical factors estimate insulin resistance in type 1 diabetes? Diabetes 200049 626-632. (doi:10.2337/diabetes.49.4.626)

26 Epstein EJ, Osman JL, Cohen HW, Rajpathak SN, Lewis O \& Crandall JP. Use of the estimated glucose disposal rate as a measure of insulin resistance in an urban multiethnic population with type 1 diabetes. Diabetes Care 201336 2280-2285. (doi:10.2337/ dc12-1693)

27 Wittmann M, Dinich J, Merrow M \& Roenneberg T. Social jetlag: misalignment of biological and social time. Chronobiology International 200623 497-509. (doi:10.1080/07420520500545979)
28 Parsons MJ, Moffitt TE, Gregory AM, Goldman-Mellor S, Nolan PM, Poulton R \& Caspi A. Social jetlag, obesity and metabolic disorder: investigation in a cohort study. International Journal of Obesity 201539 842-848. (doi:10.1038/ijo.2014.201)

29 Lemieux I, Pascot A, Couillard C, Lamarche B, Tchernof A, Almeras N, Bergeron J, Gaudet D, Tremblay G, Prud'homme D et al. Hypertriglyceridemic waist: a marker of the atherogenic metabolic triad (hyperinsulinemia; hyperapolipoprotein B; small, dense LDL) in men? Circulation 2000102 179-184. (doi:10.1161/01.CIR.102.2.179)

30 Marmot M. What kind of society do we want: getting the balance right. Lancet 2015385 1614-1615. (doi:10.1016/s01406736(15)60784-x)

31 Edwards EM, Stuver SO, Heeren TC \& Fredman L. Job strain and incident metabolic syndrome over 5 years of follow-up: the coronary artery risk development in young adults study. Journal of Occupational and Environmental Medicine 201254 1447-1452. (doi:10.1097/ JOM.0b013e3182783f27)

32 Borel AL, Benhamou PY, Baguet JP, Halimi S, Levy P, Mallion JM \& Pepin JL. High prevalence of obstructive sleep apnoea syndrome in a type 1 diabetic adult population: a pilot study. Diabetic Medicine 2010 27 1328-1329. (doi:10.1111/j.1464-5491.2010.03096.x)

33 Manin G, Pons A, Baltzinger P, Moreau F, Iamandi C, Wilhelm JM, Lenoble P, Kessler L \& Kessler R. Obstructive sleep apnoea in people with type 1 diabetes: prevalence and association with micro- and macrovascular complications. Diabetic Medicine 201532 90-96. (doi:10.1111/dme.12582)

34 Barone MT, Wey D, Schorr F, Franco DR, Carra MK, Lorenzi Filho G \& Menna-Barreto L. Sleep and glycemic control in type 1 diabetes. Archives of Endocrinology and Metabolism 201559 71-78. (doi:10.1590/2359-3997000000013)

35 Pullman RE, Roepke SE \& Duffy JF. Laboratory validation of an in-home method for assessing circadian phase using dim light melatonin onset (DLMO). Sleep Medicine 201213 703-706. (doi:10.1016/j.sleep.2011.11.008)

36 Keijzer H, Smits MG, Peeters T, Looman CW, Endenburg SC \& Gunnewiek JM. Evaluation of salivary melatonin measurements for Dim Light Melatonin Onset calculations in patients with possible sleep-wake rhythm disorders. Clinica Chimica Acta 2011412 1616-1620. (doi:10.1016/j.cca.2011.05.014)

37 Saxvig IW, Wilhelmsen-Langeland A, Pallesen S, Vedaa O, Nordhus IH, Sorensen E \& Bjorvatn B. Objective measures of sleep and dim light melatonin onset in adolescents and young adults with delayed sleep phase disorder compared to healthy controls. Journal of Sleep Research 201322 365-372. (doi:10.1111/jsr.12030)

38 Saxvig IW, Wilhelmsen-Langeland A, Pallesen S, Vedaa O, Nordhus IH $\&$ Bjorvatn B. A randomized controlled trial with bright light and melatonin for delayed sleep phase disorder: effects on subjective and objective sleep. Chronobiology International 201431 72-86. (doi:10.3109/07420528.2013.823200)

39 Meliska CJ, Martinez LF, Lopez AM, Sorenson DL, Nowakowski S \& Parry BL. Relationship of morningness-eveningness questionnaire score to melatonin and sleep timing, body mass index and atypical depressive symptoms in peri- and post-menopausal women. Psychiatry Research 2011188 88-95. (doi:10.1016/j.psychres.2010.12.010)

40 McMullan CJ, Schernhammer ES, Rimm EB, Hu FB \& Forman JP. Melatonin secretion and the incidence of type 2 diabetes. JAMA 2013 309 1388-1396. (doi:10.1001/jama.2013.2710)

Received 1 March 2016

Revised version received 2 August 2016

Accepted 16 August 2016 